\title{
Grupos de acogimiento: Sistematización de un modelo participativo para la formulación diagnóstica y terapéutica en un centro de salud mental comunitaria. Chorrillos-Perú, 2018.
}

Foster groups: Systematization of a participatory model for the diagnostic and therapeutic formulation in a community mental health center. Chorrillos-Peru, 2018.

Humberto Castillo-Martell ${ }^{1,2, a}$, Nestor Carrasco-Montoya ${ }^{3, b}$, Amalia Valdivia-Quintanilla ${ }^{3, b}$, Catiuska Zambrano-Leon ${ }^{3, c}$, Laura Perez-Arce ${ }^{3, c}$, Elizabeth Pariona-Ruiz ${ }^{3, d}$, Cintya Mendoza-Alberca ${ }^{3, d}$, Alida Caycho-Valle ${ }^{3, b}$, Cristina Ojeda-Nieto ${ }^{3, c}$.

\section{RESUMEN}

Objetivo: Sistematizar el proceso de formulación de diagnósticos y planes de tratamiento en grupos participativos de profesionales y usuarios, denominados Grupos de Acogimiento, como alternativa al modelo convencional de consultas psiquiátricas individuales y secuenciales, en el ámbito de la salud mental comunitaria. Material y Métodos: El estudio se realizó en un centro de salud mental comunitaria (CSMC) de Lima durante el periodo enero-diciembre de 2018. Se siguió una metodología de investigación-acción en la que propuestas surgidas de la experiencia se implementaban para, luego, analizar críticamente sus resultados y ajustarlos sucesivamente hasta lograr un punto de estandarización o de consenso entre los participantes. Resultados: El modelo participativo se aplicó en $97 \%$ de los usuarios durante el periodo de observación y se llegó a la formulación de diagnósticos y planes de tratamiento durante la sesión del Grupo de Acogimiento, en el 85\% de los pacientes. Las sesiones se estandarizaron en cuatro momentos concatenados y semi-estructurados: Presentación horizontal de participantes, comprensión empática mutua, exploración colectiva de causas, y decisiones terapéuticas compartidas. Conclusiones: El modelo participativo de Grupos de Acogimiento fue una alternativa viable y eficaz para la formulación inicial de diagnósticos y planes terapéuticos en el CSMC, con fases semiestructuradas convenientemente estandarizadas y replicables.

PALABRAS CLAVE: Servicios de salud mental, salud comunitaria, diagnóstico psiquiátrico.

\section{SUMMARY}

Objective: To systematize the process of diagnosis and treatment plan formulations in participatory groups of users and professionals, called Fostering Groups, as an alternative to the conventional model of individual and sequential psychiatric consults, in the field of community mental health. Material and Methods: The study was carried

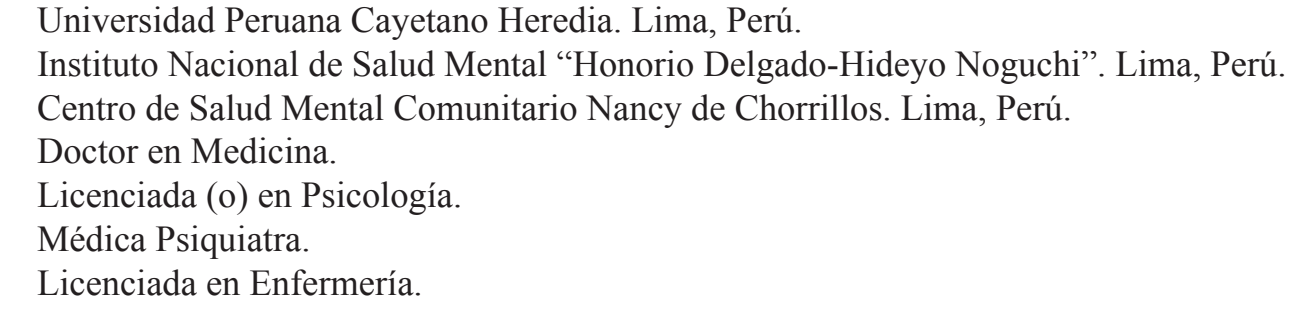


out in a community mental health center (CMHC) in Lima throughout the period January-December 2018. An action-research methodology was followed, in which proposals arising from the experiences were put into action to, then, critically analyze the and adjust them successively until reaching a point of standardization or consensus among the participants. Results: The participatory model was applied in $97 \%$ of the users during the observation period and the formulation of diagnoses and treatment plans during the Foster Group session was successfully reached in $85 \%$ of the patients. The sessions were standardized in four concatenated and semi-structured moments: Horizontal presentation of participants, mutual empathetic understanding, collective exploration of causes, and shared therapeutic decisions. Conclusions: The Fostering group model was a viable and effective alternative for the initial formulation of diagnoses and therapeutic plans in the CMHC, with semi-structured phases conveniently standardized and replicable.

KEYWORDS: Mental health services, community health, psychiatric diagnosis.

\section{INTRODUCCIÓN}

En el Perú se viene implementando una reforma de los servicios de salud mental bajo el enfoque de salud mental comunitaria basado en el desplazamiento del eje de atención de los grandes hospitales a centros de salud mental comunitarios (CSMC) que cuentan con profesionales de las diferentes disciplinas de la salud mental y se orientan al cuidado de la salud mental de poblaciones delimitadas territorialmente $(1,2)$. A finales de 2019, se habían implementado 154 de los aproximadamente 1000 que se necesitan para cubrir el territorio nacional (3). Fundamentalmente, la reforma implica el cuidado de la salud mental individual y colectiva de manera continua, bidireccional y colaborativa entre las instituciones públicas o privadas y la comunidad (4). Para esto, es necesario un cambio en los paradigmas y formas de atender y cuidar la salud mental que incluye el trabajo interdisciplinario permanente, la integración de la atención de salud mental a las prácticas generales de salud, y sobre todo, la articulación de los enfoques, saberes y recursos institucionales y comunitarios, en un marco de respeto a los derechos y equiparación de las relaciones de poder entre usuarios y proveedores del sistema $(5,6)$.

Un aspecto clave en la atención de salud, es el primer encuentro entre los proveedores de los servicios y los usuarios. En éste se instauran los estilos, condiciones, límites y posibilidades de las ulteriores relaciones y prácticas de cuidado de la salud mental (7). La atención convencional en los servicios de la salud mental suele iniciar con una entrevista de enfermería donde el paciente expone por primera vez su problemática para ser derivado a una consulta psicológica o psiquiátrica (8), donde nuevamente relata la situación y a través de una entrevista, el profesional formula diagnósticos o hipótesis explicativas de la situación, y a partir de ello, un plan de tratamiento de acuerdo a su especialidad y competencias (9).

Los enfoques de salud mental comunitaria cuestionan los diagnósticos convencionales y más bien, se proponen la formulación de casos que se expresan como relatos descriptivos, comprensivos y explicativos de los procesos mentales y contextuales problemáticos, con la finalidad de implementar intervenciones biológicas, psicológicas o sociales para reducir el sufrimiento, mejorar la calidad de vida y/o restablecer la salud mental de las personas, familias y comunidades (10). La formulación de casos de forma interdisciplinaria y participativa -también llamada diagnósticos comunitarios- generalmente requiere de sesiones de trabajo adicionales entre los profesionales que analizaron la situación del paciente desde sus respectivas disciplinas, y en otras, de una sesión interdisciplinaria con el paciente y sus acompañantes. Estas modalidades demandan mucho tiempo y esfuerzo de coordinación por lo que se limitan principalmente a casos complejos.

En el presente artículo, se presenta la sistematización del proceso de formulación de diagnósticos y planes de tratamiento en los Grupos de Acogimiento (pequeños grupos participativos de usuarios y profesionales), como alternativa al modelo convencional de consultas individuales y secuenciales que predominan en los servicios de salud mental, realizada en un centro de salud mental comunitario en Lima, Perú.

\section{MATERIAL Y MÉTODOS}

El presente estudio se llevó a cabo en el Centro de Salud Mental Comunitario Nancy Reyes ubicado en el distrito de Chorrillos, en Lima-Perú. Las unidades de observación son todos los Grupos de Acogimiento 
Grupos de acogimiento: Sistematización de un modelo participativo para la formulación diagnóstica y terapéutica.

implementados durante el año 2018. De los 392 grupos que se realizaron en este periodo, 162 estuvieron enfocados en adultos, 98 en adolescentes y 132 en niños.

Se siguió una metodología de investigación acción basada en la recursividad de experiencia-reflexión participativa-acción (11). La experiencia inicial se generó en la rutina de presentarse y dar la bienvenida a los usuarios y familiares que acudían por primera vez en búsqueda de atención. En estas reuniones los usuarios, en lugar de sentirse perturbados por la presencia de otros, tendían a explayarse en sus relatos, y parecían estimularse mutuamente. Los profesionales notaron que con la información que recibían se podían formular hipótesis de diagnóstico y trabajo. De esta manera, los Grupos de Acogimiento fueron desplazando a las convencionales entrevistas o consultas secuenciales de enfermería, trabajo social, psicología, psiquiatría y farmacia. La organización y dinámica de las sesiones se fue construyendo y ajustando colaborativamente con propuestas basadas en fundamentos teóricos y experiencias de los investigadores, que se ponían en acto para ser luego analizadas críticamente y ajustadas sucesivamente hasta lograr un punto de estandarización o de consenso entre los participantes.

Todas las sesiones de grupos contaron con la participación de al menos un miembro del equipo de investigación, quienes además de cumplir un rol en la dinámica del grupo, realizaron observaciones y anotaciones sobre los procesos y variables de análisis. Los profesionales que participaron en las sesiones de los Grupos de Acogimiento, se reunieron cada semana para compartir, sistematizar y retroalimentar los procesos.

\section{RESULTADOS}

Durante el periodo de estudio se admitieron al CSMC 1864 usuarios nuevos para recibir atención en su primera cita; $32 \%$ fueron personas referidas de centros de salud del primer nivel y $68 \%$ concurrieron de modo espontáneo y por iniciativa propia. De estos 1808 pacientes $(97 \%)$ su admisión al centro fue a través de los Grupos de Acogimiento. En 56 casos no fue posible la inclusión por decisión del paciente o por recomendación de los profesionales. El tiempo promedio de espera entre la solicitud de la cita y la atención en el grupo fue de 6 días.

La duración de las sesiones al inicio del periodo de observación variaba entre 90 y 150 minutos, luego se fue estandarizando a un promedio de 120 minutos convirtiéndose en el tiempo de referencia para los participantes. El número de participantes variaba de 3 a 6 pacientes (excepcionalmente 7) junto a sus acompañantes. Al respecto, el número de referencia asociado a un mejor rendimiento del grupo se fue definiendo en cuatro pacientes. Adicionalmente, en caso de adultos, participaba un acompañante por decisión del usuario, lo cual ocurrió en en el $72 \%$ de los casos. En las sesiones con niños y adolescentes, estos fueron acompañados por la madre (65\% de casos), por el padre (15\% de casos), por ambos $(10 \%$ de casos) y por otros cuidadores ( $10 \%$ de casos).

La participación de profesionales fue variable en los grupos, entre 3 y 6 profesionales de diferentes disciplinas, entre ellos, psiquiatra, psicólogo, enfermera, trabajador social, y/o químico farmacéutico. Asimismo, se consideró que en cada grupo debía participar por lo menos un psiquiatra y/o psicólogo. Fue frecuente la presencia de internos de psicología, médicos residentes $\mathrm{y}$ en ocasiones observadores invitados.

Durante el proceso de investigación-acción, las sesiones se fueron estandarizando en cuatro fases bien delimitados y semi estructurados con las siguientes características que en el tercio final del periodo de observación se repetían regularmente (tabla 1).

\section{Presentación horizontal de participantes}

La actividad en el grupo se iniciaba con la ratificación de la participación voluntaria y el acuerdo de confidencialidad, seguidos de la presentación de todos los participantes sentados aleatoriamente. El o la moderadora se presentaba mencionando aspectos personales, tales como su edad, lugar de nacimiento, posición entre sus hermanos, personas con las que vivía, actividades comunitarias que realizaba, además de su profesión o posición en el centro, práctica que era seguida por profesionales y usuarios. El patrón de presentación era seguido con variantes por profesionales, pacientes y acompañantes y cualquier otro participante. En esta fase no se hablaba del problema que motivaba la consulta. Se observó que esta práctica permitía establecer vínculos identitarios entre participantes sobre múltiples ejes, relativizando la diferencia radical entre profesionales y usuarios que caracteriza la relación proveedor-usuario convencional. Se observó que estos y otros rituales permitían la instalación del grupo y su disposición a asumir la tarea colaborativa, cuya metodología y 
Tabla 1. Fases del modelo de Grupos de Acogimiento para la formulación diagnóstica y terapéutica en la atención de salud mental comunitaria. Chorrillos-Peru, 2018.

\begin{tabular}{|c|c|c|c|}
\hline FASE & META & $\begin{array}{l}\text { PREGUNTAS } \\
\text { MOTIVADORAS } \\
\end{array}$ & RECURSOS DE APOYO \\
\hline $\begin{array}{l}\text { Presentación } \\
\text { horizontal de } \\
\text { participantes }\end{array}$ & $\begin{array}{l}\text { Reducir barreras } \\
\text { implícitas de } \\
\text { comunicación entre } \\
\text { usuarios y profesionales }\end{array}$ & $\begin{array}{l}\text { ¿Quiénes somos, a qué } \\
\text { nos dedicamos, qué roles } \\
\text { tenemos? }\end{array}$ & $\begin{array}{l}\text {-Acuerdos de participación voluntaria, } \\
\text { confidencialidad y observancia de } \\
\text { reglas. } \\
\text {-Esclarecer misión y metodología del } \\
\text { grupo. } \\
\text { - Resaltar identidades compartidas. }\end{array}$ \\
\hline $\begin{array}{l}\text { Comprensión } \\
\text { empática } \\
\text { mutua }\end{array}$ & $\begin{array}{l}\text { Que cada paciente } \\
\text { relate auténticamente su } \\
\text { situación problemática, } \\
\text { y que los demás } \\
\text { lo comprendan } \\
\text { empáticamente. }\end{array}$ & $\begin{array}{l}\text { ¿Qué está pasando en su } \\
\text { vida o en su mente que le } \\
\text { trae a buscar ayuda en este } \\
\text { centro? }\end{array}$ & $\begin{array}{l}\text {-Pedir a las otras personas que } \\
\text { escuchen con atención e imaginen lo } \\
\text { que está viviendo el relatante. } \\
\text {-Facilitar que fluya el relato y la } \\
\text { expresión de las emociones. } \\
\text {-Limitar excesos o distorsiones. }\end{array}$ \\
\hline $\begin{array}{l}\text { Exploración } \\
\text { colectiva de } \\
\text { causas }\end{array}$ & $\begin{array}{l}\text { Que los participantes } \\
\text { exploren diversas } \\
\text { causas y construyan } \\
\text { propuestas explicativas } \\
\text { de los problemas de salud } \\
\text { mental de sí mismos y } \\
\text { los demás. }\end{array}$ & $\begin{array}{l}\text { ¿Cuáles pueden ser las } \\
\text { principales causas que } \\
\text { expliquen los problemas y/o } \\
\text { sufrimiento del paciente? }\end{array}$ & $\begin{array}{l}\text {-Usar tipología simple para } \\
\text { organizar problemática (diagnostico } \\
\text { comunitario): } \\
\text { A: Expectativas no satisfechas. } \\
\text { B: Problemas situacionales } \\
\text { C: Síntomas o trastornos mentales } \\
\text { D: Patrones relacionales "toxicos". } \\
\text { E: Condiciones que requieren } \\
\text { cuidados especiales prolongados. } \\
\text {-Hacer relatos explicativos de } \\
\text { problemática (formulación de casos) }\end{array}$ \\
\hline $\begin{array}{l}\text { Decisiones } \\
\text { terapéuticas } \\
\text { compartidas }\end{array}$ & $\begin{array}{l}\text { Seleccionar de forma } \\
\text { colaborativa un conjunto } \\
\text { de acciones terapéuticas } \\
\text { y establecer acuerdos } \\
\text { para su realización. }\end{array}$ & $\begin{array}{l}\text { ¿Qué tipo de acciones } \\
\text { biológicas, psicosociales y } \\
\text { comunitarias requerirá el } \\
\text { paciente para mejorar su } \\
\text { vida y salud mental? }\end{array}$ & $\begin{array}{l}\text {-Hacer una mención breve de } \\
\text { diagnósticos y/o hipótesis causales } \\
\text { para enmarcar propuestas. } \\
\text {-Explicar brevemente los mecanismos } \\
\text { de acción medicamentos e } \\
\text { intervenciones terapéuticas. }\end{array}$ \\
\hline
\end{tabular}

objetivos es explicada por el moderador de una manera sencilla. Se solía decir que la metodología consistía en prestarnos atención los unos a los otros, escuchar con atención e imaginar lo que las otras personas están viviendo, pensar con nuestra mayor experiencia e inteligencia las causas de los problemas y ponerse en disposición de ser ayudado y ayudar a los otros. También la consigna de participación se resumía en observar-escuchar-sentir-analizar-actuar.

\section{Comprensión empática mutua}

En esta fase, las personas secuencialmente narraban las situaciones que motivaron la demanda de atención en el centro de salud. Unos narraban situaciones problemáticas personales, familiares, laborales o sociales que les perturbaban; otros narraban la aparición o desarrollo de síntomas o malestares emocionales; otros, historias de sufrimiento a lo largo de su vida. Las narraciones eran a veces descriptivas de hechos y en muchas otras, las personas acompañaban sus relatos con expresiones emocionales como llanto, expresiones de ansiedad, enojo, tristeza, etc. Generalmente los relatos terminaban espontáneamente luego de 3 a 5 minutos. El moderador acompañaba gestual y verbalmente el relato, alentando, esclareciendo y/o limitando, en forma prudente hasta lograr tener una claridad suficiente de la problemática expresada. Se observó que la mayoría de las personas narraban con relativa facilidad situaciones íntimas como referidas a sexualidades alternativas, historias de abuso, etc. pese a observarse que habían personas que al inicio 
Grupos de acogimiento: Sistematización de un modelo participativo para la formulación diagnóstica y terapéutica.

de este momento eran muy escuetas en su expresión e inclusive algunos hacían explícita su dificultad para comunicarse en público. Fue común que las personas manifestaran haber contado mucho más de lo que pensaban y de lo que habían podido contar en sesiones individuales previas. Fueron pocas las personas en las que se percibió que elementos sustantivos para el diagnóstico fueron ocultados o no expresados.

En el caso de niños y adolescentes, los padres expresaban los motivos por el que llevaban a sus hijos al Centro. Algunos lo hacían en tono de preocupación, la mayoría en tono de crítica y muchos en forma de fastidio. La mayoría se refirió al desempeño o a conductas que consideraban inadecuadas, siendo pocos quienes hablaron de las emociones de sus hijos. Posteriormente, se escuchaba a los adolescentes o a los niños expresar (generalmente con ayuda del moderador) cómo se sentían o cómo les iba en diferentes espacios vitales (hogar, colegio). En el caso de niños, se propiciaba la realización de actividades lúdicas breves, análisis de cuentos cortos, caja de juguetes, $y / u$ otros juegos participativos, en los cuales se evidenciaron conductas y emociones congruentes y en algunos casos, contradictorias a lo expresado por los padres.

Durante esta fase se observó que se construía un ambiente de escucha y acogida capaz de recibir relatos muy dolorosos, pero también de calmar o contener comportamientos disruptivos.

Dado que los grupos eran aleatorios, la presencia de personas con síntomas psicóticos o con déficit cognitivo, o con emociones exacerbadas, ocurrió con frecuencia. Si bien se observó que existía una limitación de la empatía, la dinámica de los grupos solía continuar. En situaciones excepcionales, algunos pacientes se retiraron abruptamente de la sesión o fueron acompañados en su retiro. Durante el año de observación ningún grupo fue concluido por estos motivos.

De esta manera, en la gran mayoría de casos, la problemática, motivo de la búsqueda de atención y objeto del diagnóstico, quedó expresada en los relatos, las expresiones gestuales, la dinámica interactiva, y/o la puesta en escena a través de actividades lúdicas.

\section{Exploración colectiva de causas}

En esta parte de la sesión el tono empático y generalmente sensible del momento anterior, cambiaba a un tono más analítico y deliberativo sobre la situación problemática de cada paciente. El pedido del moderador de identificar los problemas principales de salud mental de cada paciente, desencadenaba una serie de menciones a hechos problemáticos, síntomas, y observaciones clínicamente relevantes (problemas actuales, indicios de síntomas, antecedentes familiares, antecedentes personales) expresados $\mathrm{u}$ observados por los participantes en los momentos previos. En ocasiones esto era suficiente para configurar presunciones diagnósticas pero en otras, los profesionales de diferentes disciplinas realizaban interrogatorios específicos a modo de exámenes mentales breves.

La participación de los usuarios en estas sesiones era variable. Generalmente el debate interdisciplinario de los profesionales era lo que predominaba. Cada profesional hacía énfasis en explicaciones desde las perspectivas de sus especialidades: fenómenos psicopatológicos, criterios diagnósticos de las clasificaciones internacionales, distorsiones cognitivas, interpretaciones psicoanalíticas o sistémicas, etc. En ocasiones se daban pequeños debates en relación a la primacía de unas u otras propuestas explicativas dentro de la sesión. Los usuarios se mantenían atentos a las interpretaciones y explicaciones profesionales y con frecuencia realizaban señalamientos clínicos con asertividad.

Una herramienta que permitió la convergencia de criterios, tanto de usuarios como de profesionales, fue una categorización en grandes tipos de problemas que los moderadores denominaron "diagnóstico comunitario":

Tipo A: Expectativas no satisfechas. Referida al hecho que el comportamiento o desempeño de las personas no se ajustan a normas, estándares o expectativas de sí mismos o personas influyentes de su entorno como padres, profesores, jefes o pares.

Tipo B: Problemas situacionales: Malestares o estrés asociado a situaciones personales, familiares, institucionales o comunitarias que cesarán al cambiar las condiciones externas o al adaptarse a la nueva situación.

Tipo C: Sintomas o trastornos mentales. Alteraciones desadaptativas en las formas de pensar, sentir o actuar con relativa independencia de las circunstancias, asociadas generalmente a experiencias infantiles o vulnerabilidad de las personas. 
Tipo D: Patrones relacionales "tóxicos". Alteraciones en las formas de relacionarse con otras personas que tiende a generar sufrimiento personal y de los entornos especialmente cercanos. Generalmente asociada a problemas de apego.

Tipo E: Condiciones que requieren cuidados especiales prolongados. Generalmente asociado a problemas en el desarrollo o pérdida de competencias o habilidad que limita la autonomía o el desempeño en estándares comunes.

Los participantes con relativa facilidad llegaban a establecer consensos acerca del tipo de problema en el que se situaba la mayor carga problemática de cada uno de los pacientes. Se observó que el $85 \%$ de los problemas que se encontraban en "Tipos C, D y E, habían elementos suficientes para formular diagnósticos en la categorías CIE 10, y que únicamente en un $15 \%$ se requirió una sesión complementaria individual para tal fin, generalmente más breve que la habitual.

Asimismo, se encontró que al final de esta fase, la mayoría de las personas acogían y aceptaban las formulaciones diagnósticas de su problemática, aún en casos donde es común baja conciencia de enfermedad.

\section{Decisiones terapéuticas compartidas}

Esta fase iniciaba con la pregunta del moderador "¿Qué puede hacer cada persona y/o qué podemos ofrecer desde el CSMC y la comunidad para ayudarla a superar la problemática y recuperar su salud mental?", la cual desencadenaba una serie de propuestas por parte de usuarios y profesionales. Era común observar que entre usuarios se recomendaban una serie de estrategias para superar los diferentes tipos de problemas, basadas en experiencias personales o de terceros, que incluían propuestas o recomendaciones sobre cambios personales o familiares. Era evidente que las propuestas estaban asociadas no solo a escucha empática de las problemáticas sino también a los análisis realizados en momentos previos. Muchas de ellas se hacían de forma tan directa y coloquial que hubiera sido difícil hacerlas por un terapeuta en un ambiente convencional, y sorprendía el tono amable con el que se recibían.

Los profesionales de diferentes disciplinas hacían y justificaban sus propuestas terapéuticas biológicas $\mathrm{y} / \mathrm{o}$ psicosociales basados en las formulaciones diagnósticas y explicaban brevemente el mecanismo cómo podrían estas producir cambios en el cerebro, la mente o la vida de las personas y así activar una recuperación o reducción del sufrimiento o malestares. El o la moderadora invitaba a los participantes a identificar recursos o actividades comunitarias que contribuirían al proceso de recuperación de las personas. Esto en muchas ocasiones generaba la solidaridad y la intención de colaborar mutuamente para la realización de actividades comunitarias.

Los usuarios entre sí influían mutuamente en la toma de decisiones para la aceptación o el compromiso con las diversas acciones terapéuticas, entre ellas el uso de psicofármacos. También era común que algunos pacientes proponían ser incluidos en actividades terapéuticas que eran sugeridas a otras personas. Con mayor o menor consenso de los participantes, los pacientes y familiares iban acogiendo y comprometiéndose con las propuestas formuladas por el grupo, de manera que cada usuario terminaba una combinación de acciones a realizar entre ellas el tratamiento farmacológico, actividades terapéuticas grupales y actividades comunitarias.

\section{DISCUSIÓN}

Los resultados muestran que los Grupos de Acogimiento se lograron implementar en el CSMC Nancy Reyes de Chorrillos, como una alternativa al modelo convencional de entrevistas secuenciales por distintos profesionales.

Así, bajo este modelo, los profesionales de las disciplinas de acuerdo a su objeto buscan dar cuenta de un aspecto de la problemática de salud mental, siguiendo un proceso lógico de identificación de problemas, análisis de causas y propuesta de soluciones pero de forma simultánea para un pequeño grupo de pacientes en una sola sesión. La psiquiatría pone énfasis en las mediaciones biológicas de los problemas de salud mental (12); la psicología está centrada en reconocer y explicar las conductas asociados a sufrimiento y desadaptación $(13,14)$; la enfermería se refiere principalmente a la identificación de necesidades de atención y cuidado de las personas inmersas en algún problema de salud mental (15); y el trabajo social, en los aspectos situacionales o socioeconómicos.

Los Grupos de Acogimiento, al colocar a todos los actores en el mismo escenario (16) y alternar el rol de pacientes como analizados y analistas, desencadena una multiplicidad de interacciones que 
Grupos de acogimiento: Sistematización de un modelo participativo para la formulación diagnóstica y terapéutica.

enriquecen ampliamente el proceso y rompen la pauta de dominación cognitiva (17) (sujeto-objeto) que suponen las prácticas convencionales que se buscan transformar con los enfoques de salud mental comunitaria. Al estar presentes todos los profesionales en la misma sesión, todos están expuestos al mismo relato inicial del paciente en el mismo acto, el cual suele estar cargado de mayores detalles e intensidad emocional, reduciendo el riesgo de adecuaciones o deformaciones que va sufriendo el relato del paciente en las repeticiones secuenciales. Así mismo el relato de los otros pacientes, las inquietudes de ellos por comprender y comparar sus problemáticas van ampliando y enriqueciendo la exposición de la situación problemática (18). Esto permite que la problemática se muestre en una mayor riqueza y complejidad, dando más información a los observadores de las que obtendrían en el modelo convencional (19). Los diferentes tipos de preguntas e inquietudes por parte de diferentes profesionales en el mismo acto permite que el objeto problemático -la mente- quede más expuesta -externalizada, objetivada- para ser observada (20), comprendida, interpretada y analizada.

En la práctica convencional, cada disciplina aplica sus marcos teóricos para recortar el objeto y formula sus problemas y las explicaciones causales acomodando la realidades a sus categorías de análisis (21), generalmente minimizando, desechando o haciendo irrelevante el resto de la información (22). En los Grupos de Acogimiento, la información en apariencia irrelevante para uno es considerada relevante o esencial para otro, permitiendo la complementariedad pero también, la fuerza de las interpretaciones e hipótesis más abarcativas para intentar comprender más integralmente la problemática.

Con el objetivo de estandarizar los diagnósticos en psiquiatría y salud mental siguiendo el patrón empíricoestadístico-positivista prevalente en la medicina, se ha realizado frondosas clasificaciones como más de 400 categorías que se van reajustando periódicamente por consensos profesionales $(23,24)$. Estas clasificaciones a pesar de ser ampliamente usadas tienen poco valor para orientar las acciones terapéuticas, ya que la variabilidad en los diagnósticos no tienen correlato con la variabilidad de acciones terapéuticas que por el contrario son relativamente pocas y sus variaciones obedecen a contenidos específicos o variables de contexto, que no contemplan las clasificaciones de diagnóstico $(25,26)$. En los Grupos de Acogimiento, se ha encontrado una forma de clasificar más simple -entendible por profesionales y usuarios- con fundamento psicopatológico, que logra incluir la mayor parte de la problemática expuesta por los usuarios, y que inclusive facilita la clasificación específica de los manuales de diagnóstico. Estas formulaciones, denominadas diagnósticos comunitarios, permiten una selección más racional, participativa y ética (27) de intervenciones terapéuticas, dando más protagonismo a la voz de los usuarios y sus contextos, aspectos que se revaloran desde movimientos como salud mental comunitaria, la psiquiatría crítica y la postpsiquiatría $(28,29)$.

En conclusión, el modelo de Grupos de Acogimiento fue una alternativa viable y eficaz para la formulación inicial de diagnósticos y planes terapéuticos en el centro de salud mental comunitaria y quedó estandarizado en fases semiestructuradas que permiten su replicabilidad.

Financiamiento: El estudio se financió exclusivamente con fondos personales de los autores. No se recibió apoyo económico recibido en forma de subvención, donación de equipos, aporte de medicamentos $\mathrm{u}$ otro tipo de respaldo.

Conflictos de interés: Los autores declaran no tener conflictos de interés.

\section{Correspondencia:}

Humberto Castillo Martell

Malecón Paul Harris 396 Dpto 101. Barranco, Lima, Perú.

Correo electrónico: humberto.castillo@upch.pe

Celular: 51993455698

\section{REFERENCIAS BIBLIOGRÁFICAS}

1. Miranda J, Diez-Canseco F, Araya R, et al. Transitioning Mental Health into Primary Care. Lancet Psychiatry. 2017; 4(2): 90-92. doi: 10.1016/ S2215-0366(16)30350-9

2. Castillo-Martell H, Cutipé-Cárdenas Y. Implementación, resultados iniciales y sostenibilidad de la reforma de servicios de salud mental en el Perú, 2013-2018. Rev Peru Med Exp Salud Publica. 2019; 36(2): 326-333. doi: 10.17843/rpmesp.2019.362.4624

3. Ministerio de Salud. Plan Nacional de Salud Mental COVID 19. RM 363-2020. Lima: Ministerio de Salud; 2020.

4. World Bank Group. Healing Minds, Changing Lives: A Movement for Community-Based Mental Health Care in Peru. Delivery Innovations in a Low-Income Community, 2013-2016 (2018). Washington DC: 
World Bank Group; 2018.

5. Congreso de la República. Ley 30947, Ley de Salud Mental. Lima: Diario El Peruano; 22 de mayo del 2029.

6. Ministerio de Salud. Decreto Supremo que aprueba el Reglamento de la Ley $\mathrm{N}^{\circ}$ 30947, Ley de Salud Mental.Lima: Diario El Peruano; 2020.

7. Alarcón-Guzmán R, Castillo-Martell H. The role of Community Mental Health in a new architecture of World psychiatry and Global Mental Health. Salud Ment. 2020;43(5):235-240.

8. Bjorkman T. ¿Qué es lo importante en la atención psiquiátrica ambulatoria? La calidad de la atención desde la perspectiva del paciente. Int J Qual Health Care. 1995;7(4):448-448.

9. López-Santín J. Cambios formales en la entrevista psiquiátrica: ¿Qué hay de fondo?. Rev Asoc Esp Neuropsiquiatr. 2015;35(126):277-287.

10. Duero D, Shapoff V. El conflicto nosológico en psicopatología: notas críticas sobre el diagnóstico psiquiátrico. CES Psicología. 2009; 2(2): 20-48. DOI: https://doi.org/10.21615/895

11. Colmenares E, Piñero M. La Investigación Acción. Una herramienta metodológica heurística para la comprensión y transformación de realidades $\mathrm{y}$ prácticas socio-educativas. Laurus. 2008; 14(27):96114.

12.Prvulovic D, Hampel H. ¿Un cambio de paradigma en el diagnóstico psiquiátrico moderno? Anomalías en las redes neuronales como concepto fisiopatológico y nueva herramienta de diagnóstico. Rev Psiquiatr Salud Ment. 2010;3(4):115-8.

13. Silva F. Análisis funcional de conducta y diagnóstico psicológico. Anál Modif Conduct. 1980;6(1112):347-359.

14. Nunes-Baptista M, Garcia-Capitao C, AlbaScortegagna S. Evaluación psicológica en la salud: contextos actuales. Estudios Sociales. 2006;14(28):137-161.

15. Otero-Ceballos M. Salud mental y enfermería. Rev Cubana Enfermer. 2011; 27( 4 ): 253-254.

16. Buedo P, Silberman P. Análisis Contextual-Relacional Del Sujeto: Herramienta para la Entrevista Psiquiátrica. Interdisciplinaria. 2016; 33(2):0-0.

17. Carneiro F. A Praxis da ecologia de saberes: Entrevista de Boaventura de Sousa Santos. Tempus, Actas de Saúde Colet. 2014;8(2):331.
18. Filippetti V, López M, Richaud M. Aproximación Neuropsicológica al Constructo de Empatía: Aspectos Cognitivos y Neuroanatómicos. Cuad neuropsicol. 2012; 6(1): 63-83.

19. Cabrolié M. La intersubjetividad como sintonía en las relaciones sociales: Redescubriendo a Alfred Schütz. Polis. 2010; 9(27): 317-327.

20. Castillo-Martell H. Horizontes epistemológicos de la psiquiatría: Objetivando La Subjetividad. Rev Neuropsiquiatr. 2014;77(2):78-85.

21. Carrillo A. Profesiones sanitarias y lucha de poderes en el México del Siglo XIX.Asclepio. 1998;50(2):149168.

22. Baca-Baldomero E. Reducción y Reduccionismo: Una Polémica en Psiquiatría. Rev Neuropsiquiatr. 2013;72(1-4): 25-39. DOI: https://doi.org/10.20453/ rnp.v72i1-4.1637

23. Organización Mundial de la Salud. Clasificación Internacional de Enfermedades, 10 ${ }^{\mathrm{a}}$. Ed. (CIE-10): Trastornos mentales y del comportamiento: Descripciones clínicas y pautas para el diagnóstico. Madrid: Meditor; 2010.

24. Black D, Grant J. DSM-5 TM Guidebook - The essential companion to the Diagnostic and Statistical Manual of Mental Disorders, Fifth edition (DSM5). Washington, DC: American Psychiatric Publishing; 2014.

25. Lolas F. Diagnóstico psiquiátrico: rótulo, trastorno, comorbilidad. Rev Méd Chile. 2000; 128(7):705-707.

26. Ortiz L. Relación terapéutica y tratamientos en postpsiquiatría. Rev Asoc Esp Neuropsiq. 2017; 37(132): 553-573.

27. Ramos S. Las decisiones compartidas en salud mental: Mitos, Barreras y Beneficios. Rev Psiquiatr Salud Ment. 2016; 9(3):175-176. DOI: 10.1016/j. rpsm.2016.01.003

28. Mata I, Ortiz A. Formulación de casos en salud mental: una guía de entrenamiento. En: Retolaza A, editor. Trastornos mentales comunes: Manual de orientación. Madrid: Asociación Española de Neuropsiquiatría; 2009. pp. 195-213.

29. Liberati J. La objetividad entre paréntesis: A propósito de Humberto Maturana. Utopía y prax latinoam. 2007;12(38):121-125.

Recibido: $13 / 11 / 2020$ Aceptado: 03/03/2021 